\title{
Cardioprotection-Time to Take Into Account Clinical Complexity: The Case of Antiplatelet Agents
}

\author{
Editorial to "Two Classes of Anti-Platelet Drugs Reduce Anatomical Infarct Size \\ in Monkey Hearts" by Xi-Ming Yang et al.
}

\author{
François Roubille • Jean-Claude Tardif
}

Published online: 27 January 2013

(C) Springer Science+Business Media New York 2013

Acute myocardial infarction (AMI) is a leading cause of heart failure and premature death worldwide [1]. Immediate medical treatment is required and includes antiplatelet agents such as aspirin or thienopyridines [2]. Rapid reperfusion to limit myocardial damage is also strongly recommended [2,3]. However, reperfusion has the potential to initiate additional lethal injury, known as "ischemia-reperfusion (IR) injury" and could result in increased cardiomyocyte death [4]. New therapeutic strategies that directly target the reperfusion-mediated damage have been proven to reduce infarct size in experimental animal models. These approaches include 1) ischemic postconditioning [5] (which has been shown to be effective in animals even when the postconditioning stimulus is delayed [6]); 2) pharmacological postconditioning with cyclosporine [7] or other molecules such as erythropoietin (EPO) (unpublished information); and 3) genetic perturbation in animal models of critical proapoptotic pathways involved in reperfusion injury $[8,9]$. Some of these new approaches have been shown to improve ventricular remodeling and clinical outcomes after AMI [10-13].

F. Roubille $\cdot$ J.-C. Tardif

Montreal Heart Institute, Université de Montréal,

Montreal, QC, Canada

F. Roubille

Inserm, CNRS, UMR-5203, Institut de Génomique Fonctionnelle,

INSERM, U661, Universités de Montpellier 1 and 2,

Montpellier, France

F. Roubille

Cardiology Department, University Hospital of Montpellier,

Montpellier, France

F. Roubille $(\bowtie)$

Montreal Heart Institute, 5000 Belanger Street,

Montreal, PQ H1T 1C8, Canada

e-mail: francois.roubille@gmail.com
It is difficult to dissect the molecular mechanisms mediating the beneficial effects of these promising approaches because of several confounding factors (see Fig. 1). Antiplatelet agents could be among the most powerful cardioprotective drugs. Yang et al. now provide one of the first basic studies shedding light on the role of anti-platelet agents in cardioprotection. Recently, the same authors described their initial findings in a rabbit model (in press) [14], providing mechanistic insights into the action of these anti-platelet agents. Based on the rabbit model, they proposed that the cardioprotective effects of these anti-platelet agents are not mediated by a reduction of microvascular obstruction.

In this issue of Cardiovascular Drugs and Therapy, they confirmed and report for the first time similar results in a primate model, providing convincing evidence of the cardioprotective benefits provided by antiplatelet agents following MI [15]. This is an important finding as most studies in clinical settings are hampered by the systematic use of these agents. The authors demonstrate, in a rarely used IR monkey model, a cardioprotective effect of two different classes of antiplatelet therapies: cangrelor, a powerful P2Y12 receptor inhibitor and a novel murine antibody against the collagen receptor glycoprotein VI (OM2). The antibody against the collagen receptor glycoprotein VI reacts only with the primate protein. Although the primate model has technical limitations (for example, it is difficult to dissect the molecular mechanisms mediating these beneficial effects) it also provides a reliable model for clinical translation. Both drugs provided efficient cardioprotective effects with a reduction of the infarct size/area at risk ratio between $23 \%$ and $41 \%$. This benefit is most likely mediated through the effect of the drugs on platelets, which could limit IR injury. The drugs could prevent platelet activation [16] and release of deleterious cytokines, which otherwise could have enhanced inflammation [17] or activated other cell types including 


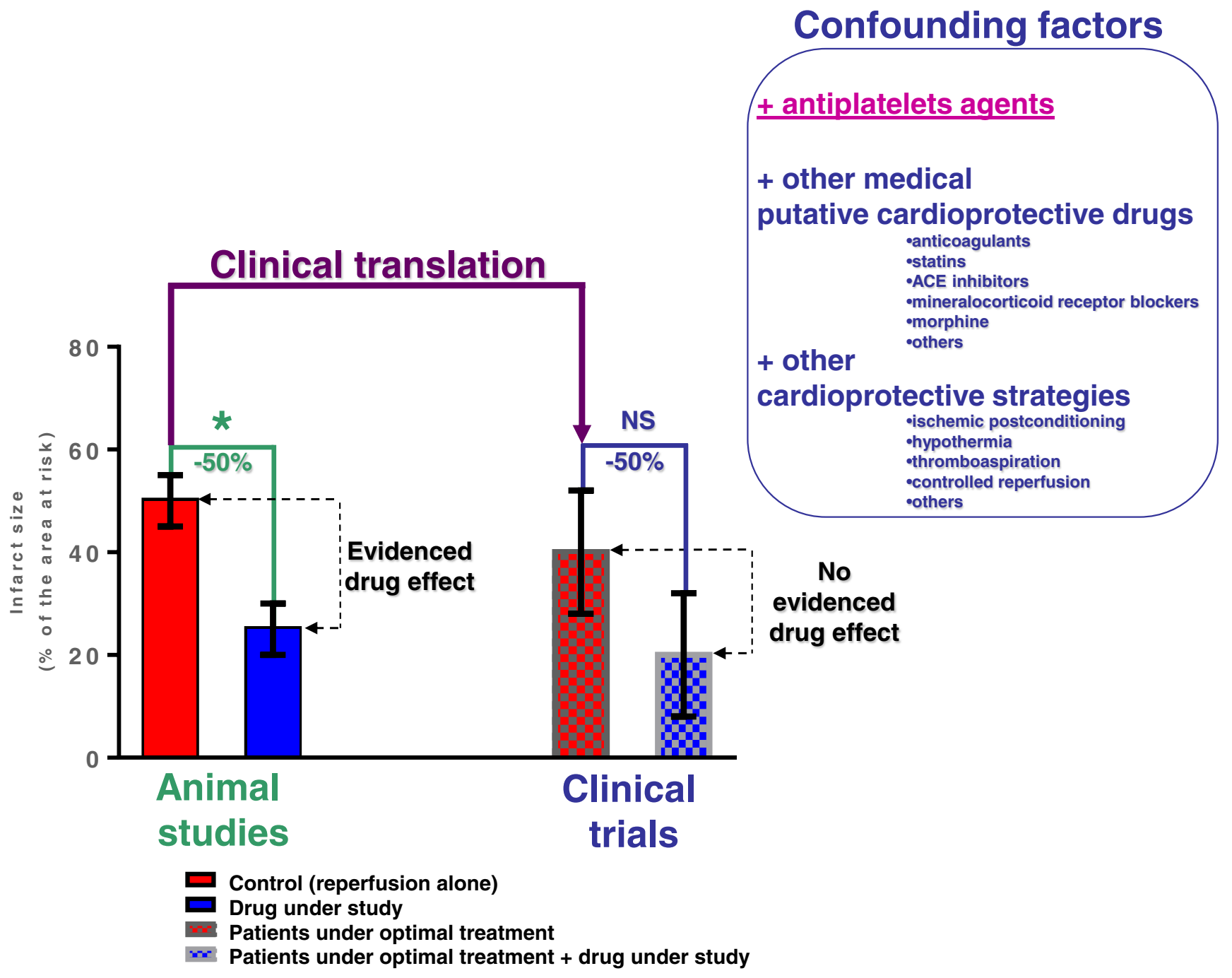

Fig. 1 Impact of cardioprotective drugs such as anti-platelet agents unheeded in the design of a clinical trial on cardioprotection, based on previous data. In animal studies on cardioprotection, infarct size (IS) after reperfusion is about $50 \%$ of the area at risk (AAR), depending on the experimental model (left panel). Many efficient cardioprotective strategies such as preconditioning, ischemic postconditioning, pharmacological postconditioning with morphine, erythropoietin (EPO), cyclosporine or others result in IS decreases of up to $50 \%$. The small variability of the effect (S.E.M is shown) combined with the large baseline AAR, large therapeutic effect, and high reproducibility contribute to the demonstration of the drug effect. By contrast, in clinical settings (right panel), baseline IS is reduced because of endogenous

leukocytes [18], endothelial cells [19] or microparticles [20]. Interestingly, the OM2 antibody offered a promising therapeutic window with an efficient anti-platelet effect and a smaller bleeding risk, when compared to cangrelor.

Despite these important findings, there are certain limitations to the model. First, a wide variability in area at risk was noted in this primate model; in contrast, this variability is not as pronounced in mouse, rat or rabbit models. This could reflect technical difficulties, such as variability of the level of artery occlusion, and/or variability among animals. cardioprotective phenomena, mainly collateral flow, as well as exogenous ones mainly other efficient unavoidable cardioprotective agents such as statins, some anti-hypertensive agents, and morphine. Antithrombotic and antiplatelet agents are the cornerstone of reperfusion with or without primary coronary intervention. Their impact could hamper the demonstration of a statistically significant effect on IS of a beneficial drug under study. Besides, clinical heterogeneity because of varying ages, comorbidities, concomitant treatments, and patient management can compound this problem and increase variability. *: The difference is statistically significant. NS: The difference is not statistically significant, although the relative risk reduction (50\%) is the same. ACE inhibitors: angiotensin-converting-enzyme inhibitors

Importantly, this observation could highlight the large variability, which is also observed in clinical settings, of ischemia duration (another important cause of clinical heterogeneity), received drugs and other clinical parameters. As noted above, it is difficult to study the mechanistic pathways in a primate model, a limitation which weakens the authors' conclusions. Finally, although outside the scope of the paper, it would have been of interest to compare a powerful cardioprotective strategy such as ischemic postconditioning to ischemic postconditioning complemented with antiplatelet agents to further 
support the notion of protection offered by antiplatelet strategies alone.

Taken together, the important findings by Yang et al. and various experimental and clinical data support a cardioprotective role of anti-platelet agents following MI. Importantly, the mechanisms of such a cardioprotective effect of antiplatelet agents, if confirmed, remain to be better understood and may provide an explanation for the large beneficial effects observed in clinical trials. With regards to clinical settings, three recent controlled, randomized, proof-of-concept studies aimed at determining whether ischemic postconditioning could limit infarct size in patients with STEMI, were retrospectively analyzed and underlined the cardioprotective effect of the antiplatelet agent clopidogrel [21]. In 88 patients, ischemic postconditioning and the anti-platelet agent clopidogrel (300$600 \mathrm{mg}$ before primary coronary intervention) were the only two additive therapeutic independent predictors of final infarct size determined by cardiac enzyme release. The cardioprotective effect was here explained by the reduction of IR injury.

Finally, all the putative cardioprotective drugs should be evaluated for their actual impact during myocardial infarction, particularly when several drugs of the same class are available, to guide our choices and treatment recommendations.

Acknowledgments This work was supported by grants from the Fédération Française de Cardiologie (FR).

\section{References}

1. WHO. WHO Fact sheet $\mathrm{N}^{\circ} 310$, updated June 2011. 2011.

2. Taylor J. 2012 ESC Guidelines on acute myocardial infarction (STEMI). Eur Heart J. 2012;33:2501-2.

3. Antman EM, Anbe DT, Armstrong PW, et al. ACC/AHA guidelines for the management of patients with ST-elevation myocardial infarction: a report of the American College of Cardiology/American Heart Association Task Force on Practice Guidelines (Committee to Revise the 1999 Guidelines for the Management of Patients with Acute Myocardial Infarction). Circulation. 2004;110:e82-292.

4. Yellon DM, Hausenloy DJ. Myocardial reperfusion injury. New Engl J Med. 2007;357:1121-35.

5. Zhao ZQ, Corvera JS, Halkos ME, et al. Inhibition of myocardial injury by ischemic postconditioning during reperfusion: comparison with ischemic preconditioning. Am J Physiol Heart Circ Physiol. 2003;285:H579-88.

6. Roubille F, Franck-Miclo A, Covinhes A, et al. Delayed postconditioning in the mouse heart in vivo. Circulation. 2011;124:1330-6.

7. Paillard M, Gomez L, Augeul L, Loufouat J, Lesnefsky EJ, Ovize M. Postconditioning inhibits mPTP opening independent of oxidative phosphorylation and membrane potential. J Mol Cell Cardiol. 2009;46:902-9.

8. Roubille F, Combes S, Leal-Sanchez J, et al. Myocardial expression of a dominant-negative form of Daxx decreases infarct size and attenuates apoptosis in an in vivo mouse model of ischemia/ reperfusion injury. Circulation. 2007;116:2709-17.

9. Vincent A, Gahide G, Sportouch-Dukhan C, et al. Downregulation of the transcription factor ZAC1 upon pre- and postconditioning protects against $\mathrm{I} / \mathrm{R}$ injury in the mouse myocardium. Cardiovasc Res. 2012;94:351-8.

10. Piot C, Croisille P, Staat P, et al. Effect of cyclosporine on reperfusion injury in acute myocardial infarction. New Engl J Med. 2008;359:473-81.

11. Staat $\mathrm{P}$, Rioufol G, Piot C, et al. Postconditioning the human heart. Circulation. 2005;112:2143-8.

12. Thibault H, Piot C, Staat P, et al. Long-term benefit of postconditioning. Circulation. 2008;117:1037-44.

13. Thuny F, Lairez O, Roubille F, et al. Post-conditioning reduces infarct size and edema in patients with ST-segment elevation myocardial infarction. J Am Coll Cardiol. 2012;59:2175-81.

14. Yang X-M, Liu Y, Cui L, et al. Platelet P2Y12 blockers confer direct postconditioning-like protection in reperfused rabbit hearts. J Cardiovasc Pharmacol Ther 2013.

15. Yang XM, Liu Y, Cui L, et al. Two classes of anti-platelet drugs reduce anatomical infarct size in monkey hearts. Cardiovas Drugs Ther 2013;27: this issue

16. Xu Y, Huo Y, Toufektsian MC, et al. Activated platelets contribute importantly to myocardial reperfusion injury. Am J Physiol Heart Circ Physiol. 2006;290:H692-9.

17. Schonberger T, Ziegler M, Borst O, et al. The dimeric platelet collagen receptor GPVI-Fc reduces platelet adhesion to activated endothelium and preserves myocardial function after transient ischemia in mice. Am J Physiol Cell Physiol. 2012;303:C757-66.

18. Diehl P, Olivier C, Halscheid C, Helbing T, Bode C, Moser M. Clopidogrel affects leukocyte dependent platelet aggregation by P2Y12 expressing leukocytes. Basic Res Cardiol. 2010;105:37987.

19. Krijnen PAJ, Hahn NE, Kholova I, et al. Loss of DPP4 activity is related to a prothrombogenic status of endothelial cells: implications for the coronary microvasculature of myocardial infarction patients. Basic Res Cardiol. 2012;107:233.

20. Montoro-Garcia S, Shantsila E, Marin F, Blann A, Lip GY. Circulating microparticles: new insights into the biochemical basis of microparticle release and activity. Basic Res Cardiol. 2011;106:911-23.

21. Roubille F, Lairez O, Mewton N, et al. Cardioprotection by clopidogrel in acute ST-elevated myocardial infarction patients: a retrospective analysis. Basic Res Cardiol. 2012;107:275. 